\title{
Intrinsic and Statistical Size Effects in Microforming
}

\author{
Tuncay Yalçinkaya ${ }^{1, a)}$, Aytekin Demirci ${ }^{1, b)}$, Igor Simonovski ${ }^{2, c)}$ and İzzet \\ Özdemir ${ }^{3, \mathrm{~d})}$ \\ ${ }^{1}$ Department of Aerospace Engineering, Middle East Technical University, Ankara 06800, Turkey. \\ ${ }^{2}$ European Commission, Joint Research Centre, Institute for Energy and Transport P.O. Box 2, NL-1755 ZG Petten, \\ The Netherlands. \\ ${ }^{3}$ Department of Civil Engineering, Izmir Institute of Technology, Urla, Izmir 35430, TURKEY

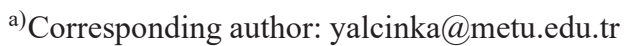 \\ b) aytekin.demirci@metu.edu.tr \\ c)Igor.Simonovski@ec.europa.eu \\ d)izzetozdemir@iyte.edu.tr
}

\begin{abstract}
This paper analyzes the intrinsic (grain size dependent) and the statistical (grain number and orientation distribution dependent) size effects of micron level polycrystalline metallic specimens under plastic deformation through a strain gradient crystal plasticity framework. The macroscopic and local behavior of specimens from very limited number of grains to high number of grains are studied and the results are discussed in detail taking into account different boundary conditions.
\end{abstract}

\section{INTRODUCTION}

The demand for the production of micro parts and therefore the improvement in the micro manufacturing technologies increased substantially in many industry clusters in the recent years. During these processes the parts with the dimensions in submillimeter scale are fabricated through micro-scaled plastic deformation. Even though there have been various classical theories developed for the modelling of macro scale deformation during conventional metal forming, it is still a challenge to establish models taking into account the size effect due to the microstructural and the statistical phenomena. Recent studies have shown that on the scale of several micrometers and below, crystalline materials behave differently from their bulk equivalent due to micro-structural effects (e.g. grain size, lattice defects and impurities), gradient effects (e.g. lattice curvature due to a non-uniform deformation field) and surface constraints (e.g. hard coatings or free interfaces). These effects could lead to stronger or weaker material response depending on the size and unique micro-structural features of the material. Moreover, as the size goes down statistical size effect comes into play as well due to the decisive behavior of individual grains which are quite limited in number.

Current paper studies both the intrinsic and the statistical size effects through a strain gradient polycrystalline plasticity framework (see [1], [2], [3], [4], [5] and [6]) for different specimen and grain sizes using Voronoi tessellation, where the polycrystalline aggregate is generated using probability theory. Applying the constitutive model to $2 \mathrm{D}$ (plane strain) polycrystalline structure, the micro tensile tests for various microstructures are numerically simulated by finite element method (FEM) in Abaqus software, where the effect of different boundary conditions is addressed as well. It is explicitly illustrated that the dominating influence of the statistical size effect fade away with the increasing number of grains.

\section{MODEL DESCRIPTION}

The constitutive model in this paper is developed as a non-convex model for deformation patterning modelling in single crystals. Here, the convex counter-part of the model is employed to model size effect prediction in metallic 
materials (see (1)). The model is based on the additive decomposition of the strain into elastic and plastic components and the plastic slip field evolution is governed by the slip law,

$$
\dot{\gamma}^{\alpha}=\dot{\gamma}_{0}^{\alpha}\left(\left|\tau^{\alpha}+\nabla \cdot \xi^{\alpha}\right| / s^{\alpha}\right)^{\frac{1}{m}} \operatorname{sign}\left(\tau^{\alpha}+\nabla \cdot \xi^{\alpha}\right)
$$

where $\tau^{\alpha}=\sigma: \mathbf{P}^{\alpha}$ is the resolved Schmid stress on the slip systems with $\mathbf{P}^{\alpha}=\frac{1}{2}\left(\mathbf{s}^{\alpha} \otimes \mathbf{n}^{\alpha}+\mathbf{n}^{\alpha} \otimes \mathbf{s}^{\alpha}\right)$, the symmetrized Schmid tensor, where $\mathbf{s}^{\alpha}$ and $\mathbf{n}^{\alpha}$ are the unit slip direction vector and unit normal vector on slip system $\alpha$, respectively and $\xi^{\alpha}$ is the microstress vector $\xi^{\alpha}=\partial \psi_{\nabla \gamma} / \partial \nabla \gamma^{\alpha}=A \nabla \gamma^{\alpha}$ bringing the plastic slip gradients into the plasticity formulation. $A$ is a scalar quantity, which includes an internal length scale parameter, and in this work it is defined as $A=E R^{2} /\left(16\left(1-v^{2}\right)\right)$ as used in [7] where $R$ is a typical length scale for dislocation interactions. In these types of models the internal length scale could be related to different microstructural features and the value would vary, e.g. [8] relates it to dislocation spacing, and [9] to grain size. In the following example we relate $R$ to a certain percentage of the grain size to study its influence. (1) is considered as one of the governing equations together with linear momentum balance which are weighted, integrated and linearized in the finite element context and implemented as a coupled user element subroutine in ABAQUS software.

\section{NUMERICAL ANALYSIS}

Voronoi tessellation is used to obtain basic geometries of the polycrystalline aggregates. In this study the aggregates contain 5, 20, 50 and 110 grains (see Fig. 1). The initial average grain size in these simulations are set to $D_{\text {avg }}=150 \mu \mathrm{m}$. Then the effect of the grain size is analyzed through a scaling operation using the internal length scale parameter, which would give values from $100 \mu \mathrm{m}$ to $375 \mu \mathrm{m}$. Note that the average grain size of AISI 316L (13 to $107 \mu \mathrm{m}$ [10]).
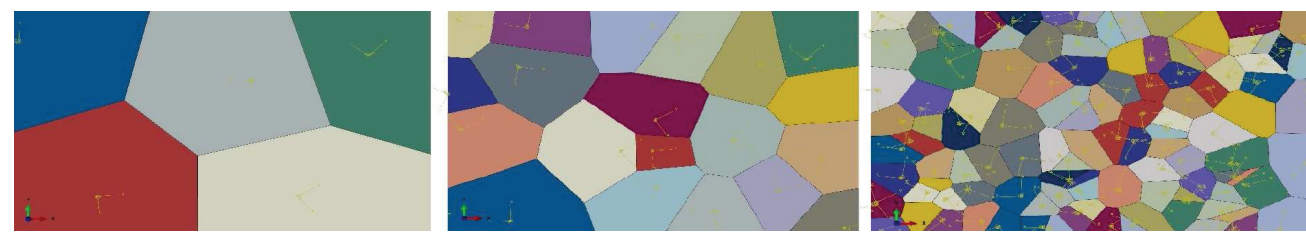

FIGURE 1: 5, 20 and 110 grain models.

Displacement load is applied to the right edge in the global $+\mathrm{x}$ direction, resulting in macroscopic $<\epsilon_{11}>=2.5 \%$. The symbol \langle\rangle represents the Macaulay bracket, indicating a macroscopically averaged value. The left edge is constrained in the $\mathrm{x}$ direction and rigid body movement is prevented by fixing the bottom left corner of the model in global y direction.

The material parameters, presented in Table 1 are not directly related to any engineering materials, rather used to demonstrate the strain gradient effects in the polycrstalline aggregates. Crystallographic orientations of grains are randomly distributed $\left(0-360^{\circ}\right)$. Two slip systems are considered in each grain.

TABLE 1: Material properties of the strain gradient crystal plasticity model.

\begin{tabular}{cccccc}
\hline $\begin{array}{c}\text { Young } \\
\text { modulus } \\
\mathrm{E}[\mathrm{MPa}]\end{array}$ & $\begin{array}{c}\text { Poisson } \\
\text { ratio } \\
v[/]\end{array}$ & $\begin{array}{c}\text { Reference } \\
\text { slip rate } \\
\dot{\gamma}_{0}\left[\mathrm{~s}^{-1}\right]\end{array}$ & $\begin{array}{c}\text { Slip } \\
\text { resistance } \\
\mathrm{s}[\mathrm{MPa}]\end{array}$ & $\begin{array}{c}\text { Orientations } \\
{\left[{ }^{\circ}\right]}\end{array}$ & $\begin{array}{c}\text { Material } \\
\text { length scale } \\
R[\mu \mathrm{m}]\end{array}$ \\
\hline 210000.0 & 0.33 & 0.05 & 20.0 & 120,60 & $1,1.5,2.5,3.75$ \\
\hline
\end{tabular}

The ratio of the average grain size to internal length scale parameter, $D_{\text {avg }} / R$ has a significant effect on the macroscopic stress-strain response, which is illustrated in Fig. 2 through equivalent stress-strain response. A small $D_{a v g} / R$ value represents a microstructure with small grain size or big internal length scale parameter, which results in a more pronounced hardening. The other advantage of the model is that the effect of the orientation distribution could explicitly be simulated. For that reason, the simulations were conducted for 20 different random orientation sets and 
the obtained results are also presented in Fig. 2. There is substantial influence of the orientation distribution on the macroscopic results. Compared to the intrinsic grain size effect due to change in the grain size, the influence of the statistical size effect is dominating for the 5 grain case.
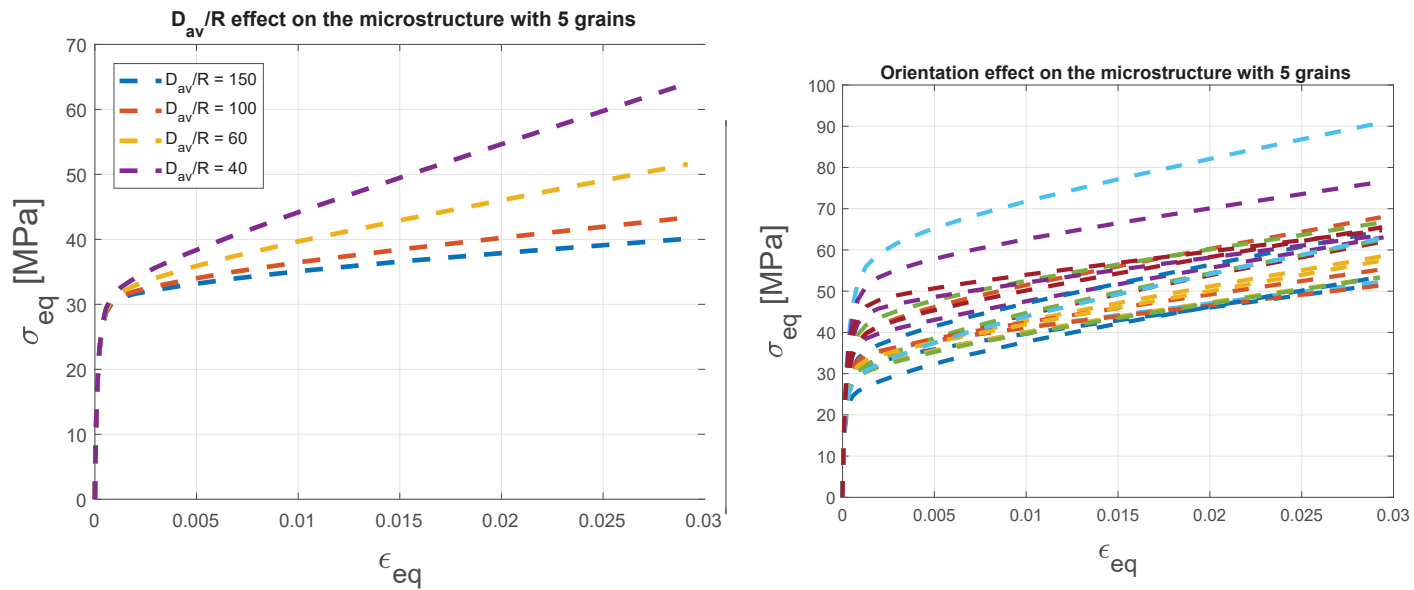

FIGURE 2: Intrinsic and statistical size effect on a microstructure with 5 grains under loading with $\dot{\varepsilon}=0.02 s^{-1}$.

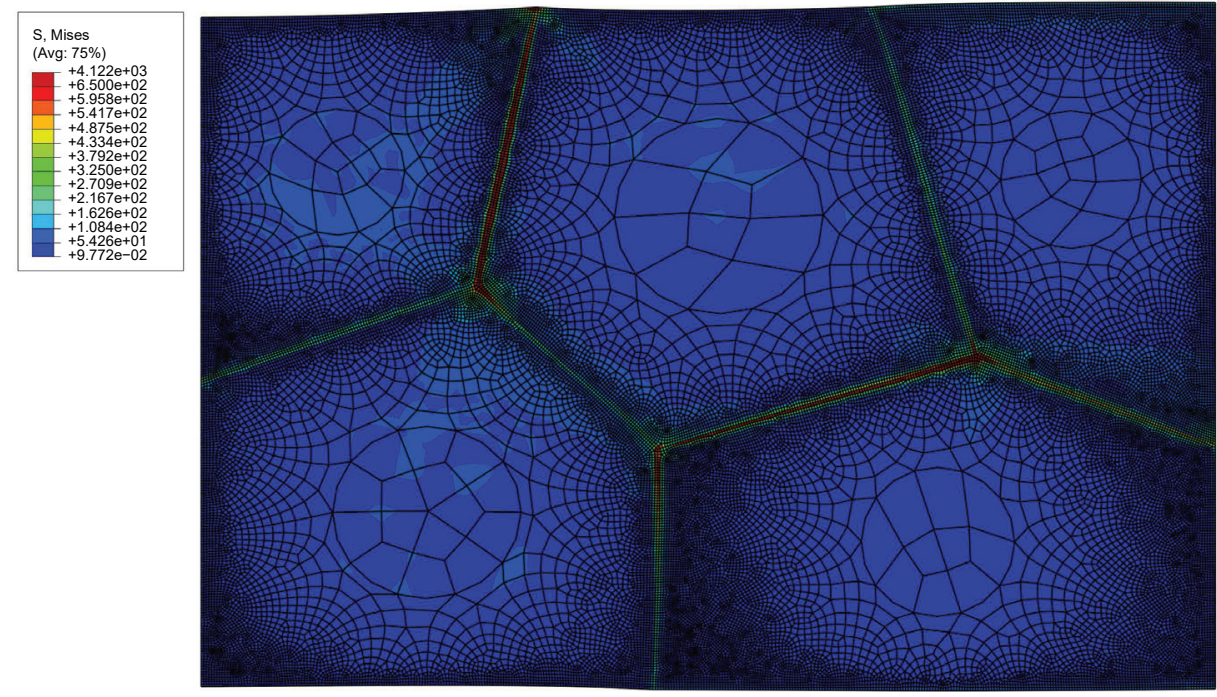

FIGURE 3: Spatial distribution of the equivalent stresses for the 5 grain microstructure.

In figures 3 and 4 the distribution of the equivalent stresses and strains are plotted respectively for the microstructure with 5 grains and for $D_{a v} / R=60$. Even though soft boundary conditions are used at the grain boundaries (the plastic slip is not restricted at the grain boundaries), the accumulation of stress and strain is observed due to the orientation difference in neighboring grains. The non-local character of the model leads to a smooth distribution of the local variables, where no sharp interface is observed which would evolve in local crystal plasticity models. Moreover the conventional (local) crystal plasticity frameworks do not include a length scale parameter therefore it is not possible to conduct this study using such models. 


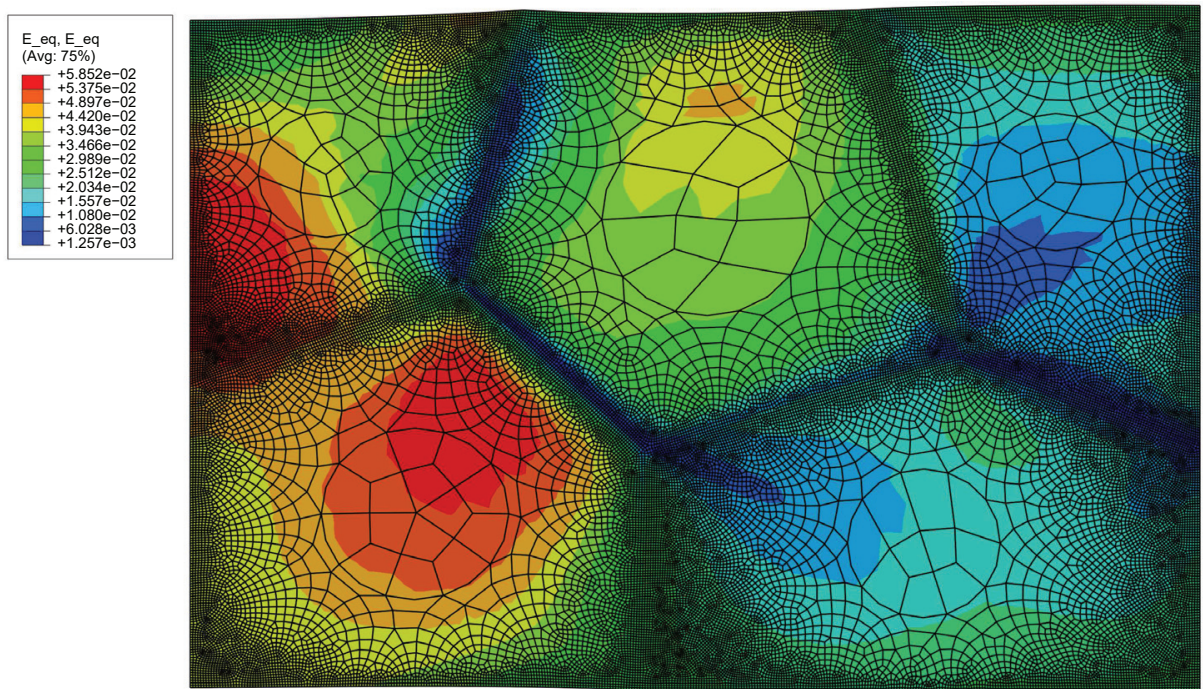

FIGURE 4: Spatial distribution of the equivalent strains for the 5 grain microstructure.
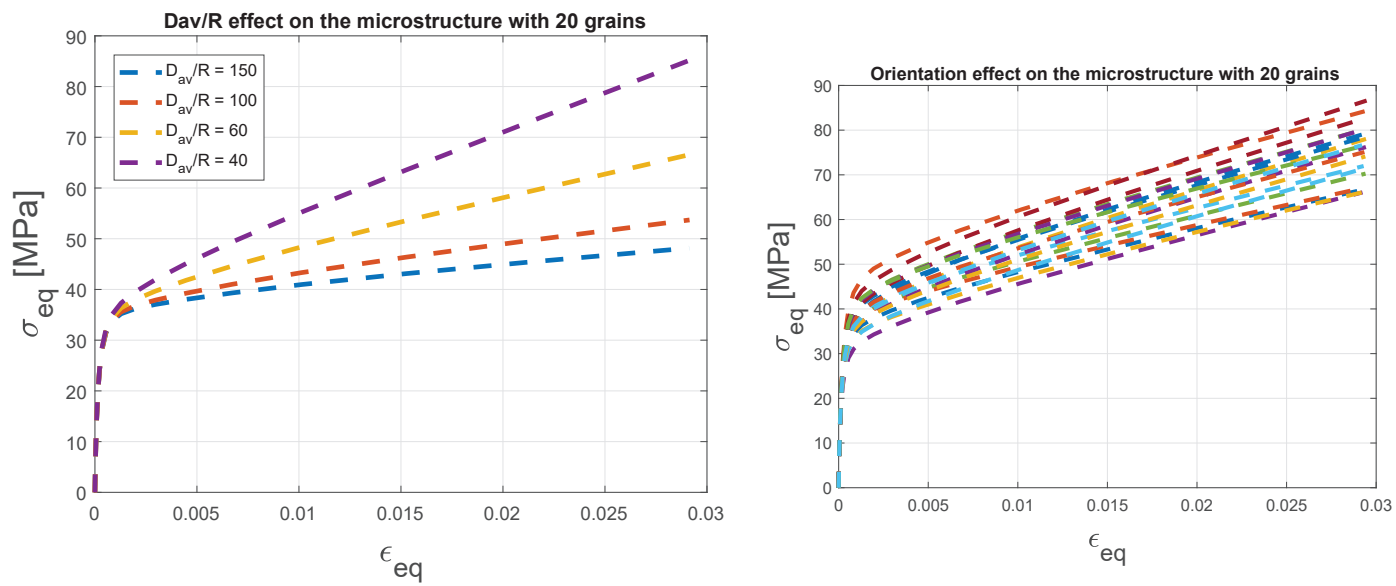

FIGURE 5: Intrinsic and statistical size effect on a microstructure with 20 grains under loading with $\dot{\varepsilon}=0.02 s^{-1}$.

It is expected that the statistical effect of random orientation distribution on the global stress-strain response would decrease with in increasing number of grains. In order to analyze this behavior with the current strain gradient crystal plasticity model, micro-structures with higher number of grains are studies in the following. First, in Fig. 5 the case with 20 grains is illustrated. In this case the effect of the $D_{\text {avg }} / R$ ratio is exactly same with the previous case, where the higher $D_{\text {avg }} / R$ value results in a weaker response. In the same figure the simulations for 20 different random orientation sets are also presented. The result shows that as the number of grains is increased the scatter in the stress-strain response is getting smaller, which is consistent with the expectations. In this microstructure the influence of grain size effect and the statistical size effect are comparable.

In Fig. 6 the same problem with 50 and 110 grains is considered where the grain boundaries have soft conditions. It is observable that as the number of grains is increased the scatter due to random grain orientations decreases. Even though there is not a pronounced difference between the two microstructure, the influence of number of grains is 

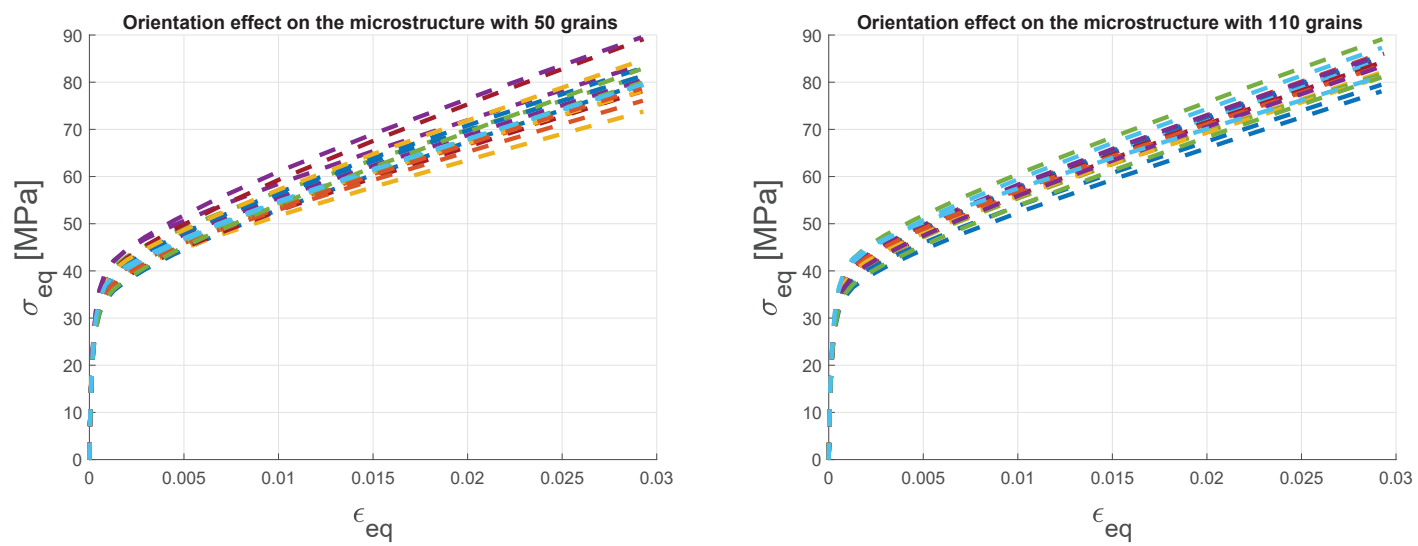

FIGURE 6: Statistical size effect on a microstructure with 50 and 110 grains under loading with $\dot{\varepsilon}=0.02 s^{-1}$.

still observable. For a more obvious difference and smaller scatter the cases with higher number of grains should be considered. After certain number of grains the material will reach the isotropic limit, which is quite expensive to illustrate with the current model.
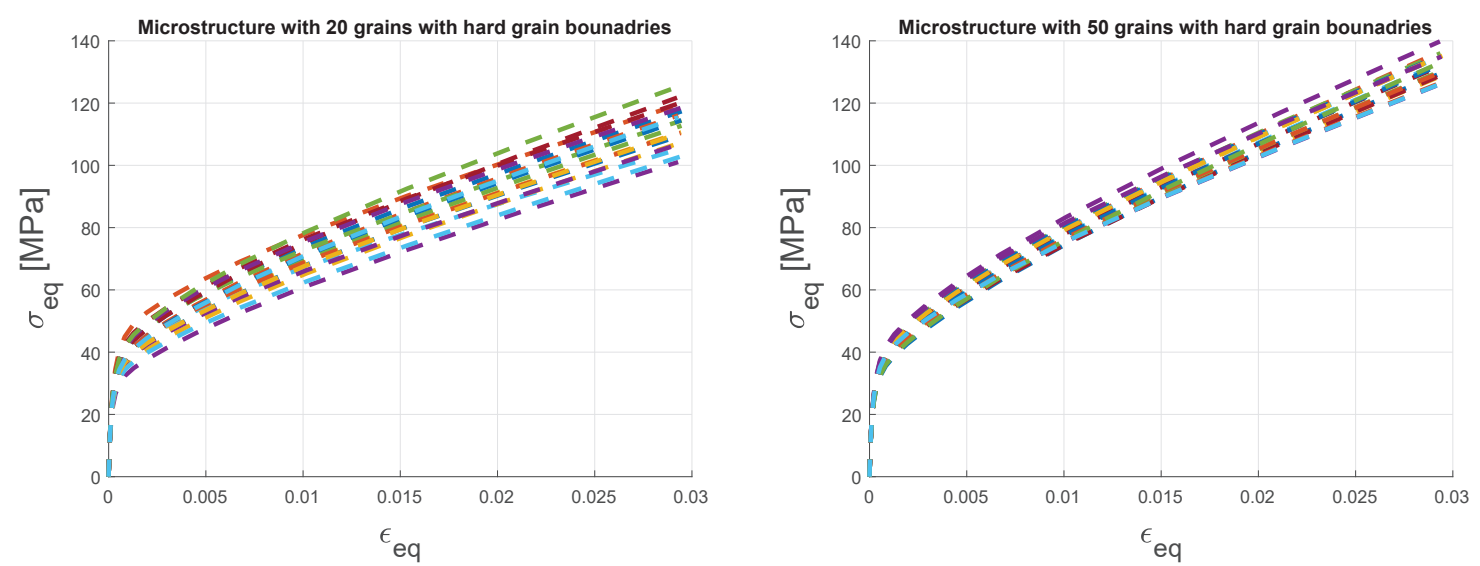

FIGURE 7: Statistical size effect on a microstructure with 20 and 50 grains under loading with $\dot{\varepsilon}=0.02 s^{-1}$ and hard grain boundaries.

In Fig. 7 influence of the hard boundary conditions on the grain boundaries is illustrated. For the 50 grains case with hard grain boundaries in Fig. 7 and soft grain boundaries in 6, the yield point of the material is not influenced much by the boundary conditions, however the hardening is affected substantially. The hard boundary case shows more hardening reaching up to $130 \mathrm{MPa}$ on average while the soft case increases up to $80 \mathrm{MPa}$ at $2.5 \%$. Another important observation in this example is that for the hard boundary case the scatter due to random grain orientation is smaller compared to soft boundary conditions. That means isotropic limit could be reached with less number of grains using the hard boundary conditions. 


\section{CONCLUSION AND DISCUSSION}

At micro level only a small number of grains are directly involved in the forming process, therefore the single grain, characterized by its individual size, orientation and position, has influence on the plasticity of the material. In order to take into account explicitly the effect of all these parameters in finite element simulations a non-local crystal plasticity model is required. This study addresses the intrinsic (grain size dependent) and the statistical (grain number, position and orientation distribution dependent) size effects in micron level polycrystalline metallic specimens under plastic deformation through a strain gradient crystal plasticity framework. In samples including very limited number of grains the statistical effect due to random grain orientation is quite dominating since the individual grain behavior plays crucial role for both local and global material response. There is huge scatter in stress-strain curve where the differences at yield point between the lowest and highest values reach up to 300 percent. As the number of grains is increased the scatter band decreases. Current study takes into account microstructure having up to 110 grains. The isotropic limit could be obtained by increasing the number of grains, which requires expensive computations. In most of the examples, soft boundary conditions are used, however it is observed that hard conditions at the grain boundaries yield not only increase in the hardening behavior and but also decrease in the scatter band due to random orientation distribution.

\section{ACKNOWLEDGMENTS}

Tuncay Yalçinkaya gratefully acknowledges the support by the Scientific and Technological Research Council of Turkey (TÜBİTAK) under the 3001 Programme (Grant No. 215M381).

\section{REFERENCES}

[1] T. Yalcinkaya, W. A. M. Brekelmans, and M. G. D. Geers, J. Mech. Phys. Solids. 59, 1-17 (2011).

[2] T. Yalcinkaya, W. A. M. Brekelmans, and M. G. D. Geers, Int. J. Solids Struct. 49, 2625-2636 (2012).

[3] T. Yalcinkaya, "Microstructure evolution in crystal plasticity : strain path effects and dislocation slip patterning," Ph.D. thesis, Eindhoven University of Technology, The Netherlands 2011.

[4] B. Klusemann and T. Yalçinkaya, Int. J. Plast 48, 168-188 (2013).

[5] B. Klusemann, T. Yalçinkaya, M. G. D. Geers, and B. Svendsen, Comp. Mater. Sci. 80, 51-60 (2013).

[6] T. Yalçinkaya, Key Eng. Mater. 554-557, 2388-2399 (2013).

[7] C. J. Bayley, W. A. M. Brekelmans, and M. G. D. Geers, Int. J. Solids Struct. 43, 7268-7286 (2006).

[8] W. D. Nix and H. Gao, J. Mech. Phys. Solids. 46, 411-425 (1998).

[9] G. Voyiadjis and R. Abu Al-Rub, Int. J. Solids Struct. 42, 3998-4029July (2005).

[10] X. Feaugas and H. Haddou, Metall. Mater. Trans. A 34A, 2329-2340October (2003). 